\title{
LIGHT MODULATION IN ELLIPTICAL-CORE LIQUID CRYSTAL FIBERS
}

\author{
T. NASILOWSKI AND T.R. WoLIŃSKI \\ Institute of Physics, Warsaw University of Technology \\ Koszykowa 75, 00-662 Warszawa, Poland
}

\begin{abstract}
An original idea of external parameters (electric field, temperature, pressure etc.) monitoring is presented. After the theoretical analysis of the light modulation in a liquid crystal and elliptical-core anisotropic fiber the latest experimental results are reported. Due to measurands-induced changes in birefringence and propagation conditions the liquid crystal and elliptical-core fiber uniting unique advantages of liquid crystals and quality of fiber technology acts as a sensing medium and can be used for continuous monitoring of different external parameters.
\end{abstract}

PACS numbers: $42.81 .-\mathrm{i}$

\section{Introduction}

Many different parameters modulating light propagation in liquid crystals have been intensively studied over the past years holding great potential for applications to various kind of sensors utilizing fiber optic sensing techniques. Any prospective liquid crystal sensors should be coupled to optical fibers that deliver optical signals to a sensing region.

Since in the functional fiber optics there is a general tendency to replace all the optical bulk elements by the equivalent fiber-optic realizations, in this paper results of experimental studies of light modulation in optical fibers with liquid crystalline cores under environmental conditions (electric field, temperature, pressure) have been reported.

The liquid crystalline-core optical fiber acts as an optically anisotropic medium characterized by an index ellipsoid and can serve as a fiber with easily controlled birefringence. However, the birefringence is equal to zero when the liquid crvstal molecules are parallel to the fiber axis, and also this is the typical structure of liquid crystal inside the hollow-core fiber. For no other reason, it was decided to use elliptical-core liquid crystal fibers where the birefringence has always non-zero value due to the non-symmetrical geometry of the core.

The paper presents preliminary theoretical studies of the optimum direction of the nematic liquid crystal director field inside elliptical-core optical fiber. Also influence of selected environmental parameters such as temperature, $\mathrm{AC}$ external 
electric field and hydrostatic pressure on the light modulation in liquid crystal fibers have been investigated experimentally. These studies have demonstrated the feasibility of using optical hollow-core fibers filled with liquid crystal to build switches, modulators and all-fiber sensors.

\section{Theoretical analysis of light propagation in elliptical-core liquid crystal fiber}

A totally new idea of external parameters monitoring using fibers with controlled birefringence due to the presence of liquid crystal is proposed. Specially drawn hollow-core fibers were filled with nematic liquid crystalline mixtures. A nematic liquid crystal confined in a cylinder (see Fig. 1) is considered. The director field configurations in the cylinder are determined by elastic torque and surface interactions. Basically, there are three types of alignment of the LC director inside the fiber: radial (homeotropic), planar, and a combination of two previous structures, the so-called escaped radial or axial geometry.

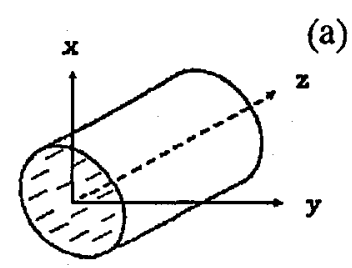

(a)
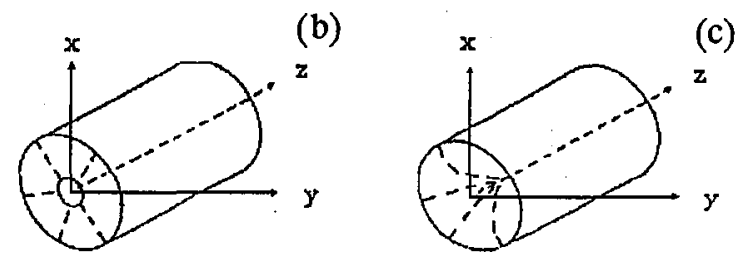

Fig. 1. Types of alignment of the LC director inside the fiber: (a) planar structure, (b) radial structure, (c) axial structure.

In theory, we start with the Maxwell equations and Frank free energy density $[1,2]$ which assumes that the orientation order of molecules is constant without changing the environmental parameters. Then the characteristic equations for the following modes: $\mathrm{TE}_{01}, \mathrm{TM}_{01}$ and $\mathrm{HE}_{11}$ are derived [3]. These formulas are called the Hondros-Debay equations and thanks to them the propagation constant $\beta$ for each of the modes can be found.

In our case, we consider both: leaky modes represented by imaginary part $\beta_{\mathrm{i}}$ and guided modes repressented by real part, $\beta_{\mathbf{r}}$. Hence a general formula describing the complex propagation constant is as follows: $\beta=\beta_{\mathrm{r}}+\mathrm{i} \beta_{\mathrm{i}}$. Then the effective refractive index $n_{\text {eff }}$ and the loss coefficient $\alpha$, which depend on components of the propagation constant can be defined

$$
n_{\text {eff }}=\frac{\beta_{\mathrm{r}}}{k} \quad \text { and } \quad \alpha=-2 \beta_{\mathrm{i}} \text {. }
$$

As it can be noticed TE and TM modes exhibit different behavior. $\mathrm{TE}_{01}$ mode is a guided mode and its propagation constant has zero imaginary component, therefore the loss coefficient disappears. On the contrary, TM modes are leaky modes, therefore their propagation constants consist of both imaginary and real part.

However, it is possible to compute numerically the propagation constant, but it is difficult to measure it experimentally and hence we cannot compare the 


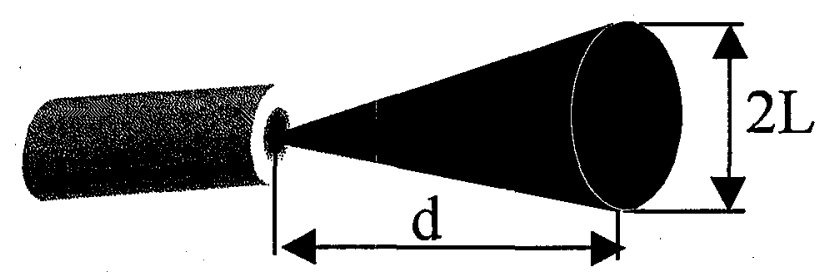

Fig. 2. Geometrical illustration for numerical aperture $(d-$ distance from fiber to the light spot and $L$ - radius of the light spot).

theory with the experiment. That is why we define the numerical aperture $N A$ (Fig. 2) that defines the divergence angle $2 \theta$ of the light outgoing from the fiber: $N A=\sin \theta$. This can be calculated from theory

$$
N A^{\text {th }}=\frac{\sqrt{k^{2}-\beta^{2}}}{k_{0}}=\sqrt{n_{\mathrm{LC}}^{2}-n_{\mathrm{eff}}^{2}}
$$

and it can be compared with the data obtained from measurements

$$
N A^{\mathrm{ex}}=\frac{L}{\sqrt{d^{2}+L^{2}}}
$$

where $k$ is the wave vector inside the fiber, $k_{0}$ is the wave vector outside the fiber (in vacuum), $d$ is the distance from fiber to the light spot, and $L$ is the radius of the light spot.

The changes of the numerical aperture influence the coupling between the liquid crystal fiber (LCF) and lead-in or lead-out fibers and they give the intensity changes in the transmitted light. We assumed that different external parameters (electric or magnetic field, temperature, hydrostatic pressure etc.) modify the numerical aperture of the LCF.

Liquid crystalline molecular ordering inside the core can be reversally modified by linear (or nonlinear) external perturbations. In this way, we obtain an externally controlled fiber with tunable birefringence and additionally, the birefringence induced inside the liquid crystal fiber is much greater comparing with "classical" silica-glass highly birefringent fibers.

An idea of investigation of the induced birefringence in the LCF leads to the fibers with elliptical liquid crystal core. First rough analysis of the birefringence has been performed utilizing the following expression from [4]:

$$
B=\Delta^{2} F(e, V) \text {, }
$$

where $\Delta=\left(n_{1}-n_{2}\right) / n_{1}, e=\sqrt{1-\left(a_{x} / a_{y}\right)^{2}}$ (ellipticity), $a_{x}, a_{y}$ - ellipse semi-axes, $V_{i}=a_{i} k_{0} n_{1} \sqrt{2 \Delta n}$ (normalized frequency), $i=x, y, k_{0}=\frac{2 \pi}{\lambda}$ and function $F$ :

$$
F(e, V)=\frac{(4 \pi)^{2}}{2 n}\left[\left(V_{x}+2\right)^{-3}-\left(V_{y}+2\right)^{-3}\right] .
$$

Figure 3; created as a result of this theory, shows the approximate changes of the birefringence of the liquid crystal fiber in function of ellipticity for ordinary and extraordinary refractive indices of LC. The effective refractive index for different LC structure can be found between the values in Fig. 3. However, in the planar 


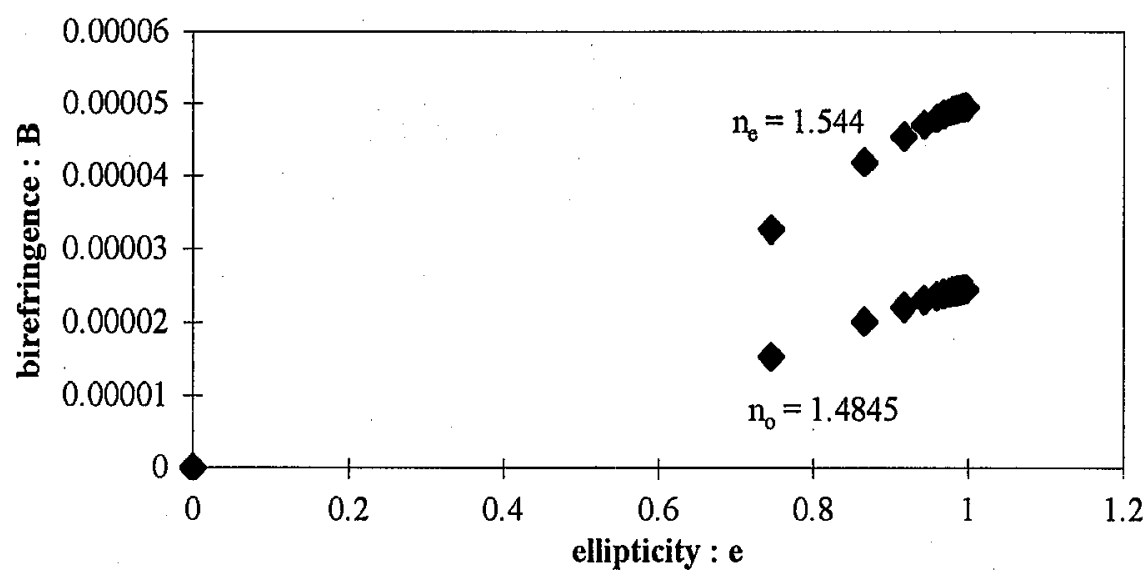

Fig. 3. Birefringence of the liquid crystal fiber in function of ellipticity of the core for ordinary and extraordinary refractive indices of LC.

structure the refractive index of the core is equal to the ordinary refractive index of the LC medium.

The more accurate theoretical analysis can be done using the method proposed in [3]. Starting from the vector wave equation

$$
\nabla_{t}^{2} \boldsymbol{E}_{t}+\left(n^{2} k^{2}-\beta^{2}\right) \boldsymbol{E}_{t}=-\nabla_{t}\left[\boldsymbol{E}_{t} \cdot \frac{1}{n^{2}} \nabla_{t}\left(n^{2}\right)\right],
$$

where $\boldsymbol{E}_{t}$ is the transverse electric field; $\nabla_{t}=\frac{\partial}{\partial x} \hat{e}_{x}+\frac{\partial}{\partial y} \hat{e}_{y}, n=n(x, y)$ and $\Delta \ll 1$ (weakly guiding approximation), we arrive at scalar wave equations for the two polarization modes ( $x$ and $y$ ):

$$
\begin{aligned}
& \nabla_{t}^{2} \phi_{x x}+\left(n^{2} k^{2}-\beta_{x}^{2}\right) \phi_{x x}=-\frac{\partial}{\partial x}\left[\frac{\phi_{x x}}{n^{2}} \frac{\partial\left(n^{2}\right)}{\partial x}\right] \quad\left(\phi_{x y} \ll \phi_{x x}\right), \\
& \nabla_{t}^{2} \phi_{x y}+\left(n^{2} k^{2}-\beta_{x}^{2}\right) \phi_{x y}=-\frac{\partial}{\partial y}\left[\frac{\phi_{x x}}{n^{2}} \frac{\partial\left(n^{2}\right)}{\partial x}\right]-\frac{\partial}{\partial y}\left[\frac{\phi_{x y}}{n^{2}} \frac{\partial\left(n^{2}\right)}{\partial y}\right]
\end{aligned}
$$

for the $x$ polarized mode, where $\boldsymbol{E}_{t}=\phi_{x x} \hat{e}_{x}+\phi_{x y} \hat{e}_{y}$ and

$$
\begin{aligned}
& \nabla_{t}^{2} \phi_{y y}+\left(n^{2} k^{2}-\beta_{y}^{2}\right) \phi_{y y}=-\frac{\partial}{\partial y}\left[\frac{\phi_{y y}}{n^{2}} \frac{\partial\left(n^{2}\right)}{\partial y}\right] \quad\left(\phi_{y y} \ll \phi_{y x}\right), \\
& \nabla_{t}^{2} \phi_{y x}+\left(n^{2} k^{2}-\beta_{y}^{2}\right) \phi_{y x}=-\frac{\partial}{\partial x}\left[\frac{\phi_{y y}}{n^{2}} \frac{\partial\left(n^{2}\right)}{\partial y}\right]-\frac{\partial}{\partial x}\left[\frac{\phi_{y x}}{n^{2}} \frac{\partial\left(n^{2}\right)}{\partial x}\right]
\end{aligned}
$$

for the $y$ polarized mode, where $\boldsymbol{E}_{t}=\phi_{y x} \hat{e}_{x}+\phi_{y y} \hat{e}_{y}$. Equation (7a) allows us to compute numerically propagation constant $\beta_{x}$ and the $x$ component of the electric field $\phi_{x x}$ for the $x$ polarized mode in the way that the sum of the electric field components over the whole cross-section of the fiber should be maximal for the proper propagation constant. Then the $y$ component of the electric field for the $x$ polarized mode can be obtained from Eq. (7b). The same numerical computation can be done for the $y$ polarized mode using Eqs. (8a) and (8b). 
Additionally, the anisotropy of liquid crystal should be implemented by. adding the dielectric tensor

$$
\bar{\varepsilon}=\left[\begin{array}{ccc}
\varepsilon_{\perp}+\Delta \varepsilon \sin ^{2} \theta & 0 & \Delta \varepsilon \sin \theta \cos \theta \\
0 & \varepsilon_{\perp} & 0 \\
\Delta \varepsilon \sin \theta \cos \theta & 0 & \varepsilon_{\perp}+\Delta \varepsilon \cos ^{2} \theta
\end{array}\right],
$$

where $\Delta \varepsilon=\varepsilon_{\|}-\varepsilon_{\perp}$ and $\theta$ is the angle between direction of molecules and the symmetry axis.

Then; the light propagation in such a fiber can be solved numerically by computing propagation constants for each polarized mode and taking into account the anisotropy of liquid crystal.

The analytical solution can be found for this case based on the wave equation in the elliptical coordinate system (Fig. 4) [5].

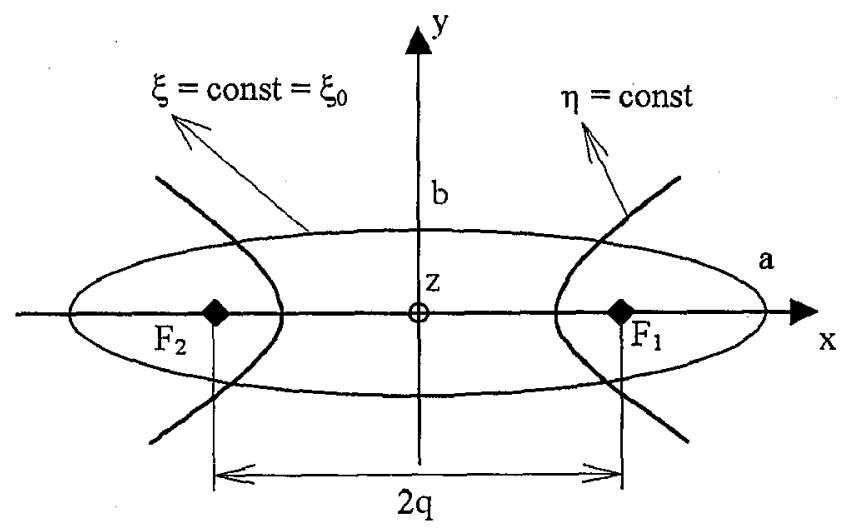

Fig. 4. Elliptical coordinate system $(\xi, \eta, z)$.

The formulae for the recalculation from the elliptical coordinates to the Cartesian coordinates are as follows:

$$
\begin{array}{ll}
x=q \operatorname{ch} \xi \cos \eta, & 0 \leq \xi \leq \infty, \\
y=q \operatorname{sh} \xi \sin \eta, & 0 \leq \eta \leq 2 \pi, \\
z=z . &
\end{array}
$$

The wave equation for the longitudinal components in the elliptical coordinate system

$$
\frac{\partial^{2} \phi_{z}}{\partial \xi^{2}}+\frac{\partial^{2} \phi_{z}}{\partial \eta^{2}}+\chi^{2} q^{2}\left(\operatorname{ch}^{2} \xi-\cos ^{2} \eta\right) \phi_{z}=0
$$

where $\phi_{z}=\left\{E_{z}, H_{z}\right\}, \chi^{2}=k_{i}^{2}-\beta^{2}, k_{i}^{2}=\omega^{2} \varepsilon_{i} \mu_{0}, i=1$ (core), 2 (cladding), gives the analytical solutions. The solutions are expressed by the infinite sum of non-modified or modified and odd or even Mathieu functions [6]. 


\section{Experiments on light modulation in LCF}

Initial experiments have been performed with circular-core liquid crystal fibers. The aim of this work was to investigate the liquid crystal fiber sensor in all-optical configuration, in which light does not leave the waveguide path (Fig. 5). Configurations of sensor with single mode fiber as a lead-in and a multimode fiber. as a lead-out were considered which exploit intensity-modulation effects.

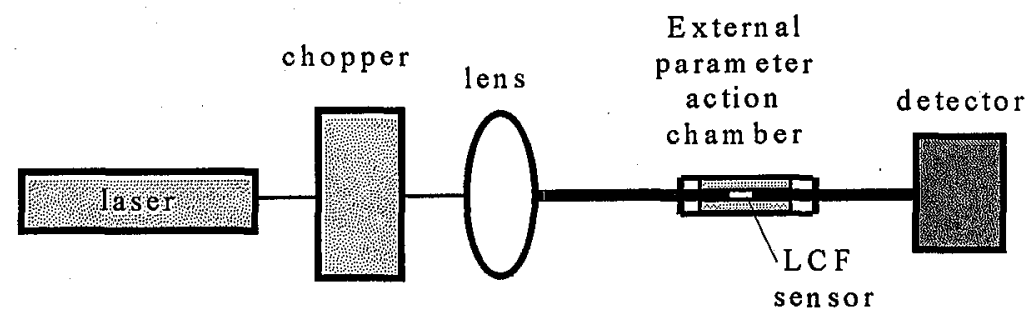

Fig. 5. Experimental setup of the fiber-optic liquid crystal sensor in all-optical configuration.

Specially drawn hollow-core fibers (capillary tubes of radii between 5 and 130 microns) were filled with a liquid crystal mixture. Another advantage of using all-fiber system is that it can stand very high pressure and can be used for measuring over $100 \mathrm{MPa}(1000 \mathrm{~atm})$ range.

It needs to be emphasized that the new method of coupling lead-in and lead-out fibers with the sensing waveguide filled up with liquid crystal was pro-. posed [7]. Such a configuration was suggested to avoid direct splicing of the liquid crystal waveguide that exhibits high thermal sensitivity.

Interesting results (Fig. 6 and Fig. 7) have been obtained for the radial and the planar configurations of the waveguide filled up with the nematic liquid crystal.

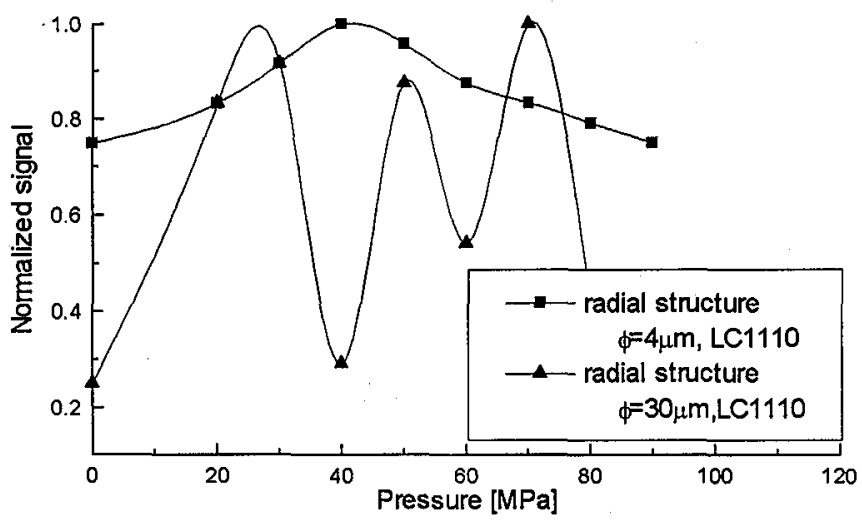

Fig. 6. Pressure characteristics of the radially oriented liquid crystal fiber with single lead-in mode fiber and a multimode lead-out fiber up to $100 \mathrm{MPa} ; 30 \mu \mathrm{m}$ and $4 \mu \mathrm{m}$ capillary filled up with nematics $(\lambda=633 \mathrm{~nm})$. 


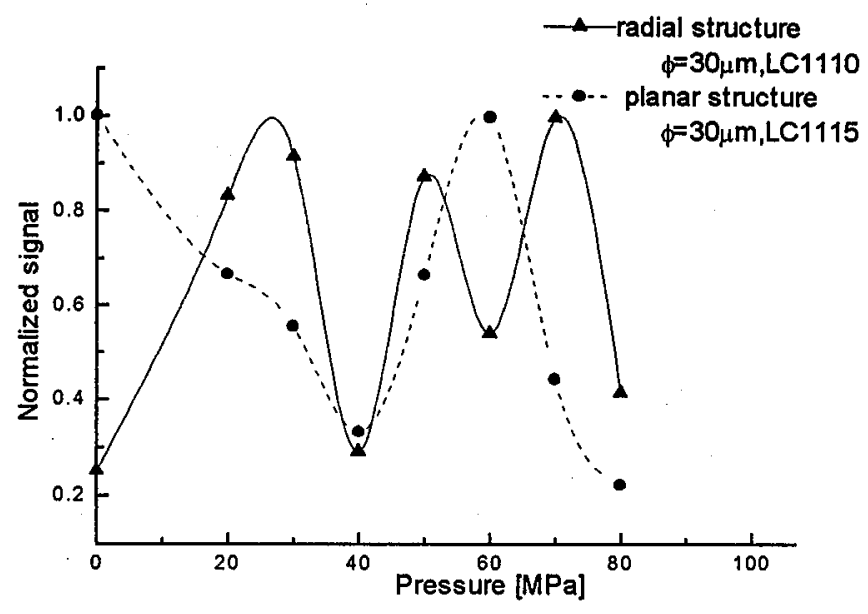

Fig. 7. Pressure characteristics of a radially and planar oriented liquid crystal fiber with a single mode lead-in fiber and a multimode lead-out fiber up to $80 \mathrm{MPa} ; 30 \mu \mathrm{m}$ capillary filled up with two different nematics $(\lambda=633 \mathrm{~nm})$.

High dynamics and the valuable repeatability of the measurements for this sensor are very important from the point of view of commercial applications. Figure 6 compares two sensors with different radius and shows better dynamics for larger radius. Also, Fig. 7 compares two sensors with different kinds and structure of LC and shows different starting point of the pressure characteristics.

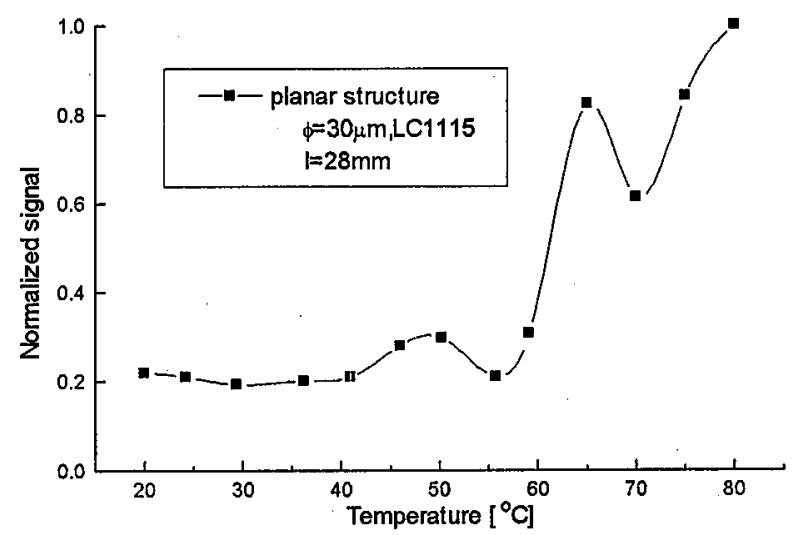

Fig. 8. Temperature characteristics of planar oriented liquid crystal fiber with lead-in single mode fiber and lead-out multimode fiber up to $80^{\circ} \mathrm{C} ; 30 \mu \mathrm{m}$ capillary filled up with nematic $(\lambda=633 \mathrm{~nm})$.

Figure 8 showing temperature characteristics, indicates the transition from the nematic to the isotropic phase between $60^{\circ} \mathrm{C}$ and $70^{\circ} \mathrm{C}$. There is a higher level of light scattering for nematic phase than with isotropic phase which gives a result in different level of the output signal. 


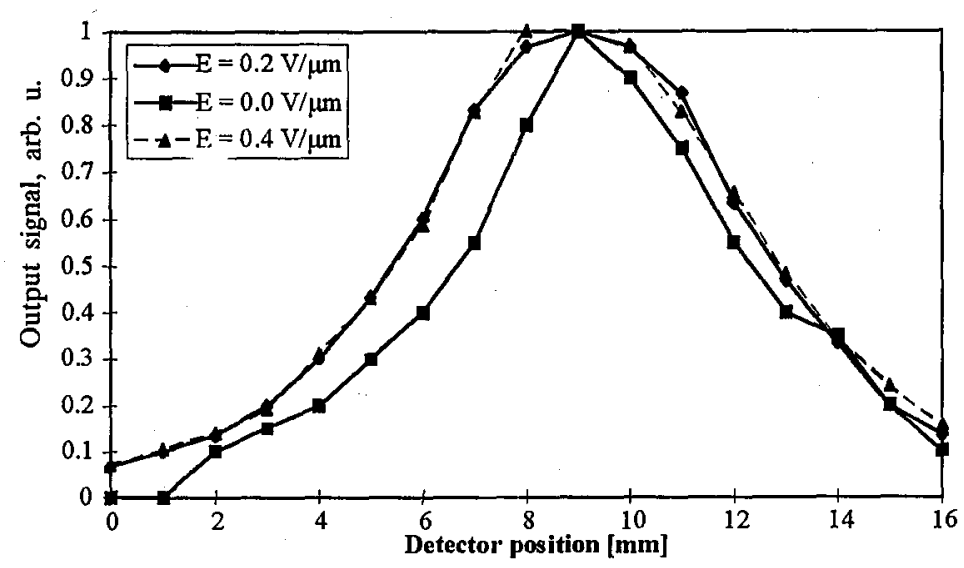

Fig. 9. Changes of numerical aperture vs. external electric field.

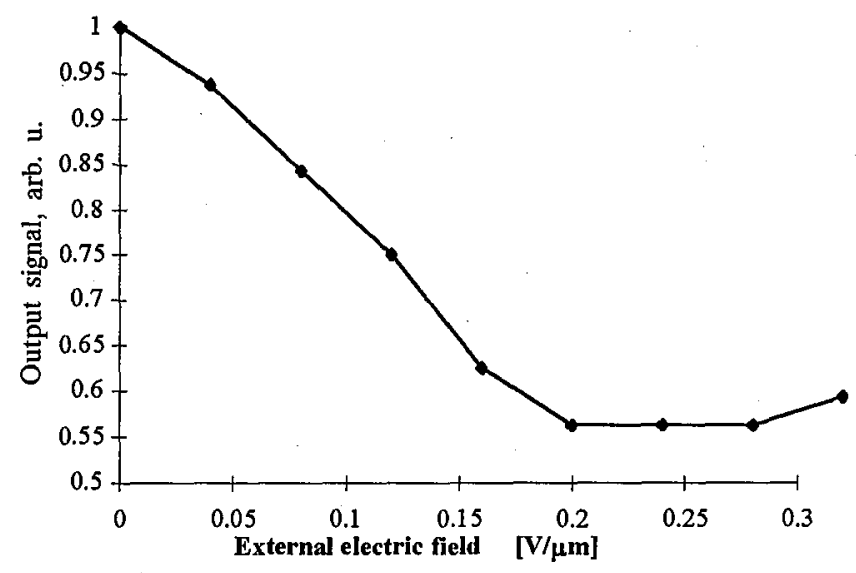

Fig. 10. Transmission of light in LCF vs. external electric field.

The changes in the numerical aperture induced by the external electric field presented in Fig. 9 influence the coupling between the liquid crystal fiber and lead-in or lead-out fibers. They result in intensity changes of the transmitted light as shown in Fig. 10. Any successful intensity LCF sensor is based on the same idea i.e. different external parameters (electric or magnetic field, temperature, hydrostatic pressure etc.) modify the numerical aperture of the LCF.

Figure 10 is only an example of the measured region of electric field, as well as other experimental figures are the examples of measured region of a given parameter.

Further experiments including also elliptical-core LCFs are still in progress.

\section{Conclusions}

The hollow-core liquid crystalline fibers with diameter 4 and $30 \mu \mathrm{m}$ have been investigated. It has been found that external electric field, hydrostatic pressure and 
temperature significantly influence the level of the optical signal propagating along the $15 \mathrm{~mm}$ elongated nematic liquid crystal-core fiber.

In the theoretical description the light propagation in elliptical-core liquid crystal fibers has been presented by three different approaches.

The principle of the intensity measurements relies on the fact that measured parameters change the numerical aperture of the liquid crystal fiber by altering the structure of molecules which influences the coupling efficiency with lead-in and lead-out fibers.

It seems to be very promising to consider full use of i.e. nonlinear effects of self-focusing in liquid crystal fiber for sensing. The external parameters could change the condition for creating such a spatial soliton, which means that by working just on the limit between linear and nonlinear effects it is possible to observe changes in the amplitude of the outgoing light.

Summarizing, the paper presents both theoretical analysis and initial experimental results for light propagation in the optical waveguides composed of LC cores and influenced by external parameters conditions. This opens up the possibility to further optimization of prospective fiber-optic liquid crystalline sensors for different parameters monitoring applications leading to a prospective construction of a compact and miniature all-fiber LC sensor. Further experiments are still in progress.

\section{Acknowledgments}

The work was supported by the Committee for Scientific Research under the research project no. 8 T11D 02: 15.

\section{References}

[1] H. Lin, P. Palffy-Muhoray, M.A. Lee, Mol. Cryst. Liq. Cryst 204, 189 (1991).

[2] T.-J. Chen, S.-H. Chen, J. Lightwave Technol. 13, 1698 (1995).

[3] K.S. Chiang, J. Lightwave Technol. LT-5, 737 (1987).

[4] M. Varnham, D.N. Payne, A.J. Barlow, R.D. Birch, J. Lightwave Technol. 1, 332 (1983).

[5] A. Majewski, Z. Krasiński, Numerical Analysis of Single-Mode Elliptical Fibers, Warsaw University of Technology Publications, Warsaw 1991.

[6] M. Abramowitz, A. Stegun, Handbook of Mathematical Functions with Formulas, Graphs, and Mathematical Tables, National Bureau of Standard Applied Mathematics Series - 55, Washington D.C. 1964.

[7] T.R. Woliński, A. Szymańska, T. Nasiłowski, M. Karpierz, A. Kujawski, R. Dąbrowski, Mol. Cryst. Liq. Cryst. 321, 113 (1998). 